\title{
Seletividade alimentar em crianças e adolescentes com transtorno do espectro autista
}

\author{
Food selectivity in children and adolescents with autism \\ spectrum disorder
}

Lilia Schug de Moraes', Vanessa Kern Bubolz², Anne y Castro Marques ${ }^{3}$ Lucia $^{\prime}$ Rota Borges ${ }^{4}$, Ludmila Correa Muniz ${ }^{5}$, Renata Torres Abib Bertacco ${ }^{6}$

${ }^{7}$ Nutricionista, Universidade Federal de Pelotas - UFPEL

${ }^{2}$ Nutricionista pela Universidade Federal de Pelotas (UFPel). Mestranda em Nutrição e Alimentos - UFPEL

${ }^{3}$ Universidade Federal de Pelotas - Doutorado em Alimentos e Nutrição pela Universidade Estadual de Campinas, Brasil (2013).

Professor Adjunto da Universidade Federal de Pelotas, Brasil.

${ }^{4}$ Universidade Federal de Pelotas, Doutorado em Ciência e Tecnologia Agroindustrial pela Universidade Federal de Pelotas, Brasil (2013). Chefe do Departamento de Nutrição Faculdade da Universidade Federal de Pelotas, Brasil.

5Universidade Federal de Pelotas - Doutorado em Epidemiologia pela Universidade Federal de Pelotas, Brasil (2015). Professora Adjunta da Universidade Federal de Pelotas, Brasil.

'Universidade Federal de Pelotas - Doutorado em Ciências Biológicas (Bioquímica) pela Universidade Federal do Rio Grande do Sul, Brasil (2011). Professora Adjunta da Faculdade de Nutrição da Universidade Federal de Pelotas, Brasil.ospital de Clínicas de Itajubá

E-mail para correspondência: Lilia Schug de Moraes - liliamoraesl@hotmail.com

\section{Resumo}

Objetivo: Caracterizar a seletividade alimentar em crianças e adolescentes com o transtorno do espectro autista (TEA). Método: Trata-se de um estudo transversal descritivo, realizado com 73 crianças e adolescentes com TEA, assistidos em um centro educacional no município de Pelotas, RS. Os dados sociodemográficos, antropométricos e de preferência alimentar foram coletados mediante anamnese, e as variáveis de seletividade alimentar foram apuradas através de um questionário, e confirmadas por meio da expressão de um ou mais domínios que compreende a seletividade: recusa alimentar, repertório limitado e alta frequência de um único alimento. Para avaliação da seletividade foi analisado um Questionário de Frequência Alimentar e três Recordatórios de 24 horas. Resultados: Da amostra avaliada, houve uma prevalência do sexo masculino (91,8\%), da cor branca (86,3\%), com média de idade de 7,1 ( $\pm 3,88)$, e com excesso de peso $(42,5 \%)$. Observou-se que a maioria $(53,4 \%)$ da amostra possuía seletividade alimentar, caracterizada principalmente pela expressão de fatores e aspectos sensoriais com base no odor dos alimentos $(56,4 \%)$, textura $(53,9 \%)$, aparência (53,8\%) e temperatura (51,3\%). Conclusão: A maioria das crianças e adolescentes com TEA avaliados demonstraram seletividade alimentar, associada a fatores sensoriais.

Palavras-chave: Comportamento Alimentar. Consumo de Alimentos Criança. Adolescente. Transtorno do Espectro Autista. 


\begin{abstract}
Objective: To characterize food selectivity in children and adolescents with autism spectrum disorder (ASD). Method: This is a quantitative, cross-sectional and descriptive study with 73 children and adolescents with ASD, male and female, attended at an educational Center in Pelotas, RS. Socio-demographic data (sex, age, color, and family income), anthropometric data and food preference were collected through nutritional anamnesis and the food selectivity variables were determined through a questionnaire of identification and characterization of selectivity. The variables were confirmed by means of the expression of one or more domains that comprise the selectivity: food refusal, limited repertoire and high frequency single food intake. To evaluate the selectivity, a Food Frequency Questionnaire and three 24-hour Reminders were analyzed. Results: Based on the analyzed sample there is a prevalence of males (91,8\%), white (86,3\%), with a mean age of 7.7 years ( \pm 3.88 ) and overweight $(42,5 \%)$. It was observed that the majority $(53,4 \%)$ of the sample had food selectivity, characterized by the expression of factors and sensorial aspects based on food odor (56,4\%), texture (53,9\%), appearance (53,8\%), and temperature (51,3\%). Conclusions: The majority of children and adolescents with ASD showed food selectivity associated with sensory factors such as texture, odor, temperature, color, taste, appearance, presentation form and brand or packaging of food.
\end{abstract}

Keywords: Eating behavior. Food Consumption. Child. Adolescent. Autistic Spectrum Disorder.

\title{
INTRODUÇÃO
}

O transtorno do espectro autista (TEA) integra um grupo de desordens do neurodesenvolvimento, que correspondem a uma série de condições que são manifestadas nos primeiros anos de vida, comprometendo o desenvolvimento típico dos indivíduos. O TEA é caracterizado por déficits persistentes, que compreende os domínios de comunicação e interação social, bem como a presença de padrões restritos, repetitivos e estereotipados, que se apresenta em diferentes contextos, relacionados ao comportamento, interesse e/ou a atividades ${ }^{1}$.

A frequência de diagnósticos de TEA tem apresentado um aumento significativo nas últimas décadas. Estima-se que, a prevalência de TEA atinja aproximadamente de 1 a cada 54 crianças $^{2}$. Existem evidências científicas que sugerem influências genéticas e ambientais como causa do TEA, porém sua etiologia ainda permanece desconhecida ${ }^{3,4}$.

Problemas com a alimentação são características marcantes e comumente descritas na conduta de crianças e adolescentes com o TEA ${ }^{5}$, evidenciadas por meio de padrões alimentares incomuns, hipersensibilidade sensorial, consumo restrito de alimentos 
e hábitos alimentares repetitivos ${ }^{6,7,8}$, geralmente associadas à seletividade alimentar. Problemas de estresse parental e desafios diários durante as refeições são relatados com frequência pelos familiares destas crianças e adolescentes, além da seletividade alimentar 9 .

A seletividade alimentar pode ser definida por um conjunto de características e aspectos variáveis, que compreende três domínios distintos, fundamentados pela recusa alimentar, por um repertório alimentar limitado e por uma ingestão alimentar específica de alta frequência habitual ${ }^{10}$. A alimentação seletiva de indivíduos com TEA é atribuída a comportamentos atípicos durante as refeições, com a expressão de algumas aversões alimentares, geralmente associadas a critérios sensoriais e impressões globais, tais como cor, textura, aparência, temperatura, odor, consistência, forma de apresentação do alimento e embalagem/marca do produto $^{11,12}$, que influenciam diretamente na escolha alimentar. Consequentemente, o comportamento alimentar de indivíduos com seletividade alimentar pode afetar a ingestão nutricional, comprometendo a qualidade da dieta, a qual contribui para o desenvolvimento de deficiências nutricionais ${ }^{13,14}$.

A primeira infância é a fase em que acontecem intensos processos de desenvolvimento ${ }^{15}$, e é durante esse período que ocorrem as primeiras experiências com a alimentação, bem como a descoberta de novos alimentos e sabores. Nesse sentido, é de extrema importância dar ênfase aos cuidados e acompanhamento nutricional, especialmente quando se trata de crianças com TEA e com seletividade alimentar. Faz-se necessário a realização de novos estudos e pesquisas que avaliem e descrevam as principais características da seletividade alimentar de indivíduos com TEA com intuito de aprimorar a intervenção dietética nesta população. Com isso, o presente estudo teve como objetivo caracterizar a seletividade alimentar em crianças e adolescentes com o transtorno do espectro autista de um centro educacional no município de Pelotas, Rio Grande do Sul.

\section{MÉTODO}

Trata-se de um estudo de natureza quantitativa do tipo transversal, com amostragem não probabilística de conveniência, realizado a partir de um recorte de dados de uma pesquisa maior, intitulada "Avaliação do Estado Nutricional de Indivíduos com Transtorno do Espectro Autista.", previamente aprovada pelo Comitê de Ética em Pesquisa da Faculdade de Medicina da Universidade Federal de Pelotas (CEP/UFPel), sob o protocolo 2.809.498. Vale ressaltar que houve liberação 
do Termo de Assentimento Livre e Esclarecido pelo CEP/UFPel, devido às características da amostra, como prejuízo nos domínios de comunicação e de interação social ${ }^{1}$.

Para a realização deste estudo, foram incluídos crianças e adolescentes com idade de 2 a 19 anos, de ambos os sexos, que receberam previamente o diagnóstico de TEA segundo o Manual de Diagnóstico e Estatística de Doenças Mentais da Academia Americana de Psiquiatria ${ }^{1}$, ou de acordo com a classificação Internacional de Doenças CID-10 16 , matriculados em um centro educacional no município de Pelotas, Rio Grande do Sul, Brasil. Foram excluídos do estudo todos os indivíduos que faziam uso de dieta restritiva intencional, e aqueles que possuíam dados incompletos na anamnese.

A coleta de dados teve início após a obtenção do Termo de Consentimento Livre e Esclarecido, assinado pelos responsáveis dos alunos participantes da pesquisa, que os acompanhavam durante o período que estavam no Centro Educacional, na sala de espera. A entrevista foi realizada com os responsáveis de forma presencial e individualizada, mediante a aplicação de quatro instrumentos de análise: Anamnese Nutricional, Questionário de Frequência Alimentar (QFA), Recordatório de 24 horas (R24h) e Questionário de Identificação e Caracterização de Seletividade Alimentar (QICSA), no período de agosto a novembro de 2018.

Primeiramente, foi aplicada a anamnese nutricional, em que constavam as variáveis sociodemográficas: sexo, cor da pele, idade e renda familiar, para caracterização da amostra, além das preferências alimentares. A variável cor da pele foi classificada como "branca" ou "não branca" autorreferida. As medidas antropométricas foram obtidas a partir da mensuração do peso, realizada em balança digital portátil da marca Tanita ${ }^{\circledR}$, com capacidade de $150 \mathrm{~kg}$, e para aferição da estatura utilizou-se uma fita métrica afixada a uma parede de superfície plana e sem rodapé, conforme preconizado pelo SISVAN ${ }^{17}$. Todas as medidas foram realizadas por pesquisadores previamente treinados.

Com base no estabelecimento dos dados antropométricos referentes ao peso e a estatura, posteriormente, realizou-se a classificação do estado nutricional dos alunos participantes, por meio do cálculo do Índice de Massa Corporal (IMC) e utilização das curvas de crescimento, seguindo os procedimentos adotados pela Organização Mundial da Saúde (OMS) ${ }^{18,19}$. O índice nutricional utilizado foi o índice de massa corporal para a idade (IMC/I). A variável de análise referente ao estado 
nutricional foi classificada em seis categorias ("magreza", "eutrofia", "risco de sobrepeso", "obesidade" e "obesidade grave"). A classificação de excesso de peso (não descrita na tabela) foi representada mediante a soma dos resultados de sobrepeso e obesidade.

Após aplicação da anamnese nutricional e da antropometria, os responsáveis foram questionados quanto à seletividade da criança ou do adolescente, através de um questionário não validado, elaborado especificamente para esta pesquisa, com a finalidade de triagem e caracterização da seletividade alimentar, intitulado "Questionário de Identificação e Caracterização de Seletividade Alimentar" (QICSA). Este questionário era constituído por 4 perguntas fechadas: se o menor apresentava seletividade alimentar ("sim" ou "não"); se apresentava recusa alimentar baseada a alguma característica sensorial ("sim" ou "não"); no caso de resposta afirmativa, qual era a frequência ("sempre" ou "às vezes") e qual era o aspecto sensorial relativo à recusa (textura, cor, sabor, odor, marca ou embalagem do produto, temperatura, forma de apresentação, aparência ou outros). Quando a resposta era positiva para a questão "outros", era questionado, de forma aberta, qual o motivo das recusas, em relação à cor, também era questionado "quais".

Para aqueles que responderam afirmativo quanto à seletividade, foram aplicados 3 R24h e 1 QFA, para a confirmação da seletividade. Para isso, foram utilizados os critérios previamente descritos por Bandini ${ }^{10}$, que são: 1) recusa alimentar (percentual de alimentos não consumidos ou percentual de alimentos recusados do que lhe foi oferecido); 2) repertório alimentar limitado (Variedade de alimentos inferior a 20, no período de 3 dias); e 3) alta frequência de consumo de um único alimento (consumo maior do que 4 a 5 vezes por dia do mesmo alimento, excluindo bebidas). A recusa alimentar foi avaliada por QFA validado, descrito a seguir, e foi considerada significativa uma recusa alimentar superior a $33 \%$ do oferecido ${ }^{20}$; o repertório alimentar limitado e a alta frequência de consumo de um único alimento foi verificado pela análise de 3 R24h. Foi considerado seletivo, o indivíduo que apresentou ao menos um destes três domínios. Cabe ainda destacar que no presente estudo houve uma adaptação ao método de Bandini ${ }^{10}$, no item referente ao repertório alimentar limitado, em que foram utilizados três recordatórios alimentares (de três dias distintos da semana), em vez de um diário alimentar de três dias, como proposto originalmente. 
O QFA respondido pelos responsáveis, referente ao consumo dos indivíduos com TEA, foi constituído por 54 itens, divididos em 9 grupos alimentares (cereais, leguminosas, vegetais, frutas, leite e derivados, carnes, gorduras, açúcares e outros). Trata-se de um questionário semi-quatitativo com perguntas fechadas, divididos em 9 categorias de frequência. No caso de resposta afirmativa para determinado item, perguntava-se a frequência de consumo (de 1 a 9 vezes), a unidade (diário, mensal ou anual), bem como o tamanho da porção consumida (pequena, média, grande ou muito grande), em relação a uma porção média de determinado alimento (apresentada em medida caseira), sendo a porção pequena equivalente à metade da porção média; a grande, o dobro da porção média; e a muito grande, duas porções médias e meia. Este instrumento foi validado previamente em uma amostra de crianças, baseado em 3 recordatórios $24 \mathrm{~h}$. Vale ressaltar que este estudo de validação ocorreu em Pelotas (resultados não publicados), mesma cidade em que ocorreu o presente estudo, e tem sido utilizado em diversas pesquisas ${ }^{21,22}$.

Os R24h foram obtidos em três dias distintos da semana (referentes a dois dias de semana e um final de semana), e respondidos pelos responsáveis dos alunos participantes.

A análise da variável de preferência alimentar foi apurada por meio do item referente aos hábitos gerais, obtido através da anamnese nutricional e que incluía duas perguntas, objetiva e subjetiva, sobre a preferência por algum alimento (Sim ou Não, Se sim, Qual?).

As variáveis categóricas foram descritas em percentuais e as variáveis contínuas, foram expressas em médias e desvio padrão. Para a associação entre as variáveis, foi utilizado o programa GraphPadPrism 7.0. Para comparação entre médias foi utilizado teste $\mathrm{t}$ de student, e para testar associações entre as variáveis categóricas, o teste do qui quadrado, considerando um nível de significância de 5\%.

\section{RESULTADOS}

Inicialmente, 77 alunos atenderam aos critérios de inclusão, porém obteve-se uma perda de 4 ao longo da pesquisa, portanto, a amostra analisada foi composta por 73 crianças e adolescentes, com média de idade 7,1 \pm 3,88 anos. A maioria eram indivíduos do sexo masculino, da cor branca e que não estavam realizando acompanhamento nutricional (Tabela 1). 


\begin{tabular}{|c|c|c|}
\hline Variável & $\mathbf{N}$ & $\%$ \\
\hline \multicolumn{3}{|l|}{ Sexo } \\
\hline Feminino & 6 & 8,2 \\
\hline Masculino & 67 & 91,8 \\
\hline \multicolumn{3}{|l|}{ Grupo etário } \\
\hline Crianças (até 10 anos) & 64 & 87,7 \\
\hline Adolescentes (>10 anos) & 9 & 12,3 \\
\hline \multicolumn{3}{|l|}{ Cor } \\
\hline Branca & 63 & 86,3 \\
\hline Não Branca & 10 & 13,7 \\
\hline \multicolumn{3}{|l|}{ Renda Familiar* } \\
\hline Não possui renda & 2 & 2,8 \\
\hline Até 1 salário mínimos & 32 & 44,4 \\
\hline De 1 a 3 salários mínimos & 28 & 38,9 \\
\hline Maior que 3 salários mínimos & 10 & 13,9 \\
\hline \multicolumn{3}{|l|}{ Estado Nutricional } \\
\hline Magreza & 1 & 1,4 \\
\hline Eutrofia & 29 & 39,7 \\
\hline Risco de Sobrepeso & 12 & 16,4 \\
\hline Sobrepeso & 9 & 12,3 \\
\hline Obesidade & 16 & 22,0 \\
\hline Obesidade Grave & 6 & 8,2 \\
\hline \multicolumn{3}{|l|}{ Acompanhamento Nutricional } \\
\hline Sim & 3 & 4,1 \\
\hline Não & 70 & 95,9 \\
\hline \multicolumn{3}{|l|}{ Seletividade Alimentar } \\
\hline Sim & 39 & 53,5 \\
\hline Não & 34 & 46,5 \\
\hline
\end{tabular}

*Renda familiar com ( $N=72$ ). Motivo: dado não contabilizado, em virtude da recusa do participante em responder o item de renda familiar.

Constatou-se que, dos 73 alunos participantes, 44 referiram seletividade alimentar, entretanto, apenas 39 destes, obtiveram a confirmação da seletividade alimentar, após avaliação dos domínios (recusa alimentar, alto consumo de um único alimento, e repertório alimentar limitado). Logo, a maioria da amostra avaliada foi composta por indivíduos seletivos (53,4\%).

Dos 39 seletivos confirmados, 59,0\% preencheram apenas um critério de seletividade, 5,1\% apresentaram 2 domínios, e 35,9\% satisfizeram os 3 domínios avaliados. A descrição dos domínios da seletividade alimentar está descrita na Tabela 2. Vale ressaltar que os indivíduos que referiram não apresentar seletividade alimentar, não tiveram os domínios avaliados. 
Quanto à classificação do estado nutricional, a maioria da amostra apresentou excesso de peso $(42,5 \%)$. Entre os indivíduos seletivos, a proporção de indivíduos com sobrepeso e obesidade manteve-se a mesma. Não houve associação significativa entre a seletividade alimentar e o estado nutricional $(P=0,5)$. Também não houve associação entre renda familiar e seletividade alimentar $(P=0,4)$.

A variável recusa alimentar foi obtida através da análise do QFA. Dos 54 itens questionados, obteve-se em média 24 alimentos recusados entre os indivíduos seletivos, que equivale a uma recusa de $44,4 \%$. Não houve diferença significativa nas médias de recusas entre as crianças e os adolescentes. Em ambos os grupos etários analisados, verificou-se que o número maior de recusas ocorreu principalmente entre os grupos alimentares de frutas e vegetais.

No tocante ao repertório alimentar, constatou-se que as crianças e adolescentes seletivas consumiam em média 17 alimentos, não havendo diferença estatisticamente significativa entre os grupos etários. Já no item de ingestão alimentar de alta frequência, apenas 5 crianças apresentaram esta característica, cujas idades variaram de 3 a 10 anos (Tabela 2).

Tabela 2 - Domínios da seletividade alimentar de crianças e adolescentes seletivas com Transtorno do Espectro Autista, alunos de um Centro Educacional Especializado do município de Pelotas/RS. Seletividade Alimentar ( $N=39)$.

\begin{tabular}{lccc}
\hline & $\begin{array}{c}\text { Crianças } \\
(\mathbf{N}=\mathbf{3 5})\end{array}$ & $\begin{array}{c}\text { Adolescentes } \\
\mathbf{( N = 4 )}\end{array}$ & $\begin{array}{c}\text { Total } \\
(\mathbf{N}=39)\end{array}$ \\
\hline $\begin{array}{l}\text { Recusa \#: } \\
\text { Média (DP) }\end{array}$ & $24,10(8,76)$ & $23,75(13,76)$ & $24,59(9,00)$ \\
$\begin{array}{l}\text { Repertório } ¥: \\
\text { Média (DP) }\end{array}$ & $16,81(5,13)$ & $18(6,24)$ & $16,7(5,12)$ \\
AFUA †: & & & \\
N(\%) & $5(14,28)$ & $0(0)$ & $5(14,28)$ \\
\hline
\end{tabular}

\#Recusa alimentar: número de itens do Questionário de Frequência Alimentar (QFA) que não foram consumidos. O QFA continha 54 itens no total.

$¥$ Repertório limitado: número de alimentos diferentes consumidos em 3 dias. Dado obtido a partir de 3 Recordatório Alimentar de 24 horas (R24h).

T Alta Frequência de um único alimento (AFUA): número de indivíduos que consumiram algum alimento mais de 4 a 5 vezes ao dia, exceto bebidas.

Quando questionados sobre a frequência em que ocorriam as recusas alimentares, dos 39 indivíduos identificados como seletivos, 28 referiram recusas "Sempre", e 11, "Às vezes". Os motivos destas recusas foram apresentados na Tabela 3. A maioria da amostra referiu que as recusas baseavam-se na textura, no odor, na temperatura e 
na aparência dos alimentos e/ou preparações. Quanto à cor dos alimentos, os mais recusados foram os verdes, verdes escuros e coloridos. Ainda, referente aos motivos das recusas, na categoria "outros", apenas 3 responsáveis responderam de forma afirmativa. As respostas para esta pergunta foram "carne vermelha", "alimentos diferentes dos habituais", e consistência de "molho", cada um destes itens foi referido por 1 participante.

\begin{tabular}{|c|c|c|}
\hline Motivos das recusas alimentares & $\mathbf{N}$ & $\%$ \\
\hline \multicolumn{3}{|l|}{ Textura } \\
\hline Sim & 21 & 53,9 \\
\hline \multicolumn{3}{|l|}{ Cor } \\
\hline Sim & 18 & 46,1 \\
\hline \multicolumn{3}{|l|}{ Sabor } \\
\hline Sim & 19 & 48,7 \\
\hline \multicolumn{3}{|l|}{ Odor } \\
\hline Sim & 22 & 56,4 \\
\hline \multicolumn{3}{|l|}{ Marca/Embalagem } \\
\hline Sim & 11 & 28,2 \\
\hline \multicolumn{3}{|l|}{ Temperatura } \\
\hline Sim & 20 & 51,3 \\
\hline \multicolumn{3}{|l|}{ Forma de apresentação do alimento } \\
\hline Sim & 18 & 46,1 \\
\hline \multicolumn{3}{|l|}{ Aparência } \\
\hline $\operatorname{sim}$ & 21 & 53,8 \\
\hline \multicolumn{3}{|l|}{ Outros } \\
\hline Sim & 3 & 7,7 \\
\hline
\end{tabular}

Com relação às preferências alimentares dos alunos participantes, a maioria $(84,9 \%)$ apresentou preferência por algum alimento específico. Os principais alimentos citados pelo grupo dos indivíduos seletivos foram: arroz, feijão, batata frita e bolachas.

\section{DISCUSSÃO}

O presente estudo identificou que as crianças e adolescentes com TEA eram na sua maioria do sexo masculino, da cor branca, apresentavam excesso de peso e não possuíam nenhum acompanhamento nutricional. Observou-se a expressão da presença de seletividade alimentar na maioria dos participantes, evidenciados 
principalmente pelos domínios de recusa alimentar e por um repertório alimentar limitado. Os principais padrões que foram identificados em relação aos fatores e aspectos sensórias que compreende a seletividade foi caracterizado com base no odor, na textura, e na aparência dos alimentos.

Com base nos resultados do presente estudo, pode-se constatar que a maioria da amostra foi do sexo masculino, corroborando com os achados de estudos de caráter epidemiológico ${ }^{23,24}$, que demonstram que a incidência de TEA é quatro vezes mais comum entre os homens, correspondendo a uma média de ocorrência de 4,6:1.

No tocante aos dados pertinentes à renda familiar mensal, foi observado, no estudo em questão, que a maioria dos indivíduos que compõem a pesquisa possuíam uma renda familiar de até 1 salário mínimo; tal achado se assemelha com um estudo ${ }^{25}$ realizado no interior de Paraíba, no qual foi evidenciado que a maioria $(80,0 \%)$ das famílias participantes possuíam uma renda média de até um salário mínimo. Verificou-se também que mais de oitenta por cento da amostra do presente estudo foi representada por indivíduos da cor branca, estes achados se assemelham com outros trabalhos ${ }^{23,26}$.

A prevalência de obesidade infantil tem crescido expressivamente nos últimos tempos ${ }^{27}$, representando um alarmante problema de saúde pública que atinge mais de um terço das crianças e adolescentes ${ }^{28}$, essa tendência mundial ocorre também entre os indivíduos com TEA ${ }^{29}$, o que implica riscos para a saúde e para o desenvolvimento ${ }^{30,31}$. Com relação ao estado nutricional, constatou-se no presente estudo, uma alta prevalência de excesso de peso, que inclui os casos de sobrepeso e obesidade. Esses dados se assemelham com outros estudos ${ }^{32,33}$. Emidio et al. avaliaram o estado nutricional de 43 crianças e adolescentes com TEA, e verificou que $13,0 \%$ apresentavam baixo peso, $21,7 \%$ estavam sobrepeso e $26,1 \%$ encontravam-se obesos. Já um estudo realizado em 2016 na capital gaúcha avaliou 49 crianças e adolescentes com TEA, e verificou que (40,0\%) dos participantes estavam em estado de sobrepeso e obesidade.

É importante ressaltar que os métodos utilizados para a avaliação antropométrica não permitem a avaliação de forma mais precisa quanto à composição corporal dos indivíduos participantes, uma vez que este é um viés inerente ao método do IMC. Entretanto, a aplicação desta ferramenta em estudos epidemiológicos ainda é o método mais prático e barato, que fornece maior facilidade de mensuração e aplicabilidade 
Acredita-se que o excesso de peso esteja condicionado a seletividade alimentar descrita, e que a questão da baixa renda socioeconômica nessa população, possa influenciar diretamente nos hábitos e escolhas de consumo alimentar desses indivíduos, pautados pela dificuldade de acesso e disponibilidade.

De acordo com alguns estudos, crianças e adolescentes com TEA podem apresentar maior frequência de sobrepeso e obesidade 29,34 . Este fato pode ser atribuído ao comportamento alimentar restrito e repetitivo, que pode influenciar diretamente no consumo alimentar, incluindo a qualidade da alimentação, pela ingestão frequente e repetitiva de alimentos não saudáveis e de elevado valor energético, favorecendo assim, o excesso de peso ${ }^{10,30}$.

Um estudo recente, realizado no Oriente Médio, comparou as principais características clínicas e comportamentais de 163 crianças e adolescentes com TEA, e 212 com desenvolvimento típico (DT), no qual não foi evidenciado diferenças significativas na média do IMC $(\mathrm{p}=0,81)$ entre os dois grupos analisados. Quanto ao comportamento alimentar dos indivíduos com TEA, foi demonstrado uma maior resistência para experimentar novos alimentos, além de problemas na forma de apresentação dos pratos e uma variedade limitada em relação o consumo de alimentos ${ }^{35}$.

Padrões alimentares inadequados são comumente relatados entre indivíduos com TEA $^{36}$. Gray et al. ${ }^{37}$ investigaram o comportamento alimentar de 31 crianças com TEA, durante as refeições, e constataram que $48,0 \%$ dos possuíam resistência para experimentar novos alimentos, 46,0\% das crianças não permaneciam sentadas à mesa até o final das refeições, e 54,2\% preferiam alimentos com consistência "crocante".

Problemas relacionados à alimentação são frequentemente descritos entre os indivíduos com TEA, e podem ser atribuídas a mecanismos correlacionados com problemas gastrointestinais, disfunções sensoriais e a inabilidade motora oral ${ }^{88,39,40}$. A conduta alimentar desses indivíduos atribuída a seletividade alimentar, é considerada um dos principais motivos de problemas alimentares durante as refeições. Todas estas condições somadas, torna o indivíduo com TEA mais predisposto ao excesso de peso e carências nutricionais.

O presente estudo examinou que a maioria das crianças e adolescentes com TEA demonstraram domínios de seletividade alimentar. As recusas alimentares ocorreram principalmente para os seguintes grupos alimentares: vegetais e frutas. 
Um estudo $^{20}$ realizado nos Estados Unidos com 54 indivíduos com TEA comparou a composição dietética entre crianças seletivas e não seletivas, e constatou que 33\% dos participantes possuíam um consumo alimentar restrito com repertório menor ou igual a 20 alimentos, representado por um consumo menor de frutas e vegetais. Além disso, os indivíduos seletivos apresentaram um consumo dietético inadequado quando comparado com o outro grupo.

Somente cinco participantes apresentaram um consumo alimentar de um único alimento maior do que 4 ou 5 vezes ao dia. Esse resultado corrobora com os achados descritos na literatura, no qual também não foi identificado associação de expressão de seletividade referentes ao item de ingestão alimentar de alta freqüência ${ }^{10}$.

Não foi observado diferença significativa quanto à presença de seletividade alimentar entre as diferentes idades analisadas. Um estudo longitudinal realizado com 52 crianças com TEA, examinou a alterações no nível de seletividade alimentar verificando a relação da super-responsividade sensorial em dois pontos do tempo representados como (tempo 1 e tempo 2) e averiguou a associação entre a seletividade alimentar e o comportamento restritivo e repetitivo no segundo tempo (tempo 2), no qual não foi identificado nenhuma mudança significativa de seletividade entre os dois tempos analisados, e foi verificado uma significativa relação entre a super-responsividade sensorial e a seletividade alimentar ${ }^{41}$.

O presente estudo evidenciou que as 39 crianças e adolescentes que obtiveram a confirmação da variável de seletividade alimentar, recusavam consumir os alimentos com base em aspectos e fatores sensoriais. As descrições características referentes ao perfil alimentar dos seletivos foram identificadas com base na textura, no odor, na aparência, na temperatura, no sabor, na cor, na forma de apresentação e marca/embalagem dos alimentos. Esses resultados corroboram com achados anteriores $^{11}$. Um estudo realizado na Itália em 2015, com 158 participantes investigou as principais características comportamentais de crianças e adolescentes com TEA, seletivos ( $n=79)$ e não seletivos ( $n=79)$; os resultados evidenciaram que o grupo de crianças seletivas demonstraram associações com fatores sensoriais, sendo as principais aversões encontradas para os itens "textura" em 68,4\%, e "sabor" em $53,2 \%$ da amostra.

As principais preferências alimentares identificadas entre o grupo de indivíduos seletivos foram: arroz, feijão, batata frita e bolacha, o que corrobora com achados de Attlee et al. ${ }^{42}$, que identificaram, em seu estudo, que os alimentos de maior 
preferência citados entre as crianças e os adolescentes com TEA foi o amido (55,8\%), e o grupo de menor preferência foi o das proteínas (32,6\%).

O presente estudo apresentou resultados consistentes, com um tamanho amostral considerável comparado a outros estudos clínicos com este público-alvo. Algumas limitações podem ser citadas, tais como o instrumento de avaliação de consumo e a falta da utilização de outros métodos para a avaliação da composição corporal, que permitisse distinguir a distribuição de massa magra e massa gorda por exemplo, além das medidas antropométricas realizadas (peso e altura). Embora este seja um viés inerente ao IMC, a utilização do índice IMC/idade é uma ferramenta bem aceita em estudos clínicos e epidemiológicos e permitiu comparar o estado nutricional da presente amostra com demais estudos da literatura. Outra limitação importante foi a ferramenta de triagem dos indivíduos seletivos utilizada, que por não ter sido validada anteriormente, pode ter subestimado o real número de indivíduos seletivos da amostra, porém, mesmo com este viés foi possível identificar um número expressivo de indivíduos com esta característica.

É importante destacar que os resultados obtidos no estudo em questão, não devem ser generalizados para outras populações, visto que se tratou de uma amostra de conveniência, e não necessariamente reflete a realidade da população com TEA em geral. Contudo, o estudo em questão representa relevante contribuição científica, fornecendo resultados importantes que abrangem a caracterização dos aspectos sensoriais da seletividade alimentar, além disso, os dados encontrados viabilizam subsídios para a elaboração e desenvolvimento de materiais educativos mais específicos, voltados às particularidades alimentares desse público. Ainda, ressaltase a importância da realização de novos estudos acerca da temática para a construção de medidas de intervenção efetivas. Como perspectivas deste trabalho, está a comparação das características de consumo entre indivíduos com TEA com e sem seletividade alimentar.

\section{CONCLUSÃO}

Diante do exposto, pode-se concluir que as crianças e adolescentes com Transtorno do Espectro Autista, na sua maioria demonstraram seletividade alimentar, evidenciada principalmente pela recusa alimentar e por possuir um repertório limitado de alimentos. 
Os principais motivos de recusa identificados foram: a textura, o odor, a temperatura e a aparência dos alimentos. Nesse contexto, em que os hábitos e escolhas alimentares podem sofrer a influência da seletividade alimentar, o acesso a um acompanhamento e orientações nutricionais individualizadas, são de extrema importância.

Mais estudos fazem-se necessários para caracterização da seletividade alimentar de indivíduos com TEA, visto que, ainda são poucas as contribuições cientificas que compreendem os aspectos gerais da seletividade alimentar.

\section{Conflito de interesse}

Não houve conflito de interesses por parte dos autores. Não houve suporte financeiro para a pesquisa.

\section{Agradecimento}

À equipe do Centro de Atendimento o Autista Dr. Danilo Rolim de Moura, ao grupo de pesquisa NUTEA, as colegas Eduarda de Souza Silva e Laura Moreira Goularte pela assistência pessoal na coleta de dados, e a todos os responsáveis dos alunos que se dispuseram participar voluntariamente da pesquisa.

\section{REFERÊNCIAS}

1. American Psychiatric Association. American. Manual Diagnóstico E Estatístico De Transtornos Mentais 5a Edição Dsm-5 ${ }^{\circledR}$. 2014. 645-667 p.

2. Report MW. Prevalence of Autism Spectrum Disorder Among Children Aged 8 Years - Autism and Developmental Disabilities Monitoring Network, 11 Sites, United States, 2016. 2020;69(4):1-12.

3. Hebert MR, Russo JP, Yang S, Roohi J, Blaxill M, Kahler SG.; Cremer L.; Hatchwell E. Autism and environmental genomics. Neurotoxicology. 2006; 27(5):671-684.

4. Mello, Ana Maria S. Ros de. Autismo: guia prático. 8 ed. São Paulo: AMA; Brasília: CORDE, 2007. 110 p.: il.

5. Ledford JR, Gast DL. Feeding Problems in Children With Autism Spectrum Disorders: A Review. Focus On Autism And Other Developmental Disabilities. 2006; 21(3):153-166.

6. Bandini LG, Curtin C, Philips S, Anderson SE, Maslin M, Must A. Changes in food selectivity in children with autism spectrum disorder. J Autism Dev Disord. 2017;47(2): 439-446.

7. Chistol LT, Bandini LG, Must A, Philips S, Cermak SA, Curtin C. Sensory Sensitivity and Food Selectivity in Children with Autism Spectrum Disorder. J Autism Dev Disord. 2017; 48(2):583-591. 
Lilia Schug de Moraes, Vanessa Kern Bubolz, Anne y Castro Marques, Lucia Rota Borges, Ludmila Correa Muniz, Renata Torres Abib Bertacco | Seletividade alimentar em crianças e adolescentes com transtorno do espectro autista

8. Stough CO, Gillette MLD, Roberts MC.; Jorgensen TD, Patton SR. Mealtime Behaviors Associated with Consumption of Unfamiliar Foods by Young Children with Autism Spectrum Disorder. Appetite. 2015;95: 324-333.

9. Thullen M, Bonsall A. Co-Parenting Quality, Parenting Stress, and Feeding Challenges in Families with a Child Diagnosed with Autism Spectrum Disorder. J Autism Dev Disord. 2017;47(3):878886.https://doi.org/10.1007/s10803-0162988-X

10. Bandini LG, Anderson SE, Cermak S, Evans EW, Scampini R, Maslin M, Must A. Food Selectivity in Children with Autism Spectrum Disorders and Typically Developing Children. The Journal of Pediatrics. 2010;157(2):259-264.

11. Postorino V, Sanges V, Giovagnoli G, Fatta LM, De Peppo L, Armando M, et al. Clinical differences in children with autism spectrum disorder with and without food selectivity. Appetite [Internet]. 2015[acesso em 2018 Out 12];92:126-32. Disponível em: http://dx.doi.org/10.1016/j.appet.2015.05.016

12. Schrec KA, Williams K, Smith AF. A Comparison of Eating Behaviors Between. Journal of Autism and Developmental Disorders. 2004;34(4):433-8.

13. Carvalho JÁ, Santos CSS, Carvalho MP, Souza LS. Nutrição e autismo: considerações sobre a alimentação do autista. Revista Científica do ITPAC. 2012;5(1):1-7.

14. Ranjan S, Nasser JA. Nutritional Status of Individuals with Autism Spectrum Disorders: Do We Know Enough?. Advances in Nutrition. 2015;6(4):397-407.

15. Bundy DAP, de Silva N, Horton S, Patton GC, Schultz L, Jamison DT. Investment in child and adolescent health and development: key messages from Disease Control Priorities, 3rd Edition. The Lancet. 2017;391(10121):687-699. doi: 10.1016/S0140-6736(17)32417-0.

16. Organização Mundial de Saúde. Classificação estatística internacional de doenças e problemas relacionados à saúde (Kortmann): CID-10. 10.ed. São Paulo; 2000. p.361-362.

17. Ministério da Saúde (BR), Coordenação Geral da Política de Alimentação e Nutrição. Vigilância Alimentar e Nutricional: SISVAN. Orientações para a coleta e análise de dados antropométricos em serviços de saúde. Norma Técnica SISVAN. Material Preliminar. Brasília: Ministério da Saúde; 2008.

18. . Organização Mundial da Saúde - OMS. Growth reference data for 0-5 years, 2006. Disponível em: http://www.who.int/childgrowth/standars/bmi. [Acesso em 20 out 2018].

19. Organização Mundial da Saúde - OMS. Growth reference data for 5-19 years, 2007. Disponível em:

http://www.who.int/growthref/who2007_bmi_for_age/en/. [Acesso em 20 out 2018].

20. Curtin C, Hubbard K, Anderson SE, Mick E, Must A, Bandini LG. Food Selectivity, Mealtime Behavior Problems, Spousal Stress, and Family Food Choices in Children with and without Autism Spectrum Disorder. J Autism Dev Disord. 2015;45(10):3308-3315

21. Santos LP, Assunção MCF, Matijasevich A, Santos IS, Barros AJD. Dietary intake patterns of children aged 6 years and their association with socioeconomic and 
Lilia Schug de Moraes, Vanessa Kern Bubolz, Anne y Castro Marques, Lucia Rota Borges, Ludmila Correa Muniz, Renata Torres Abib Bertacco | Seletividade alimentar em crianças e adolescentes com transtorno do espectro autista

demographic characteristics, early feeding practices and body mass index. BMC Public Health (2016) 16:1055.

22. Leal KK, Schneider BC, França GVA, Gigante DP, Santos I, Assunção MCF. Qualidade da dieta de pré-escolares de 2 a 5 anos residentes na área urbana da cidade de Pelotas, RS. Rev. paul. pediatr. [online]. 2015, vol.33, n.3, pp.310-317. ISSN 1984-0462. https://doi.org/10.1016/j.rpped.2015.05.002.

23. Fombonne E. Epidemiology of Autistic Disorder and Other Pervasive Developmental Disorders. J Clin Psychiatry. 2005;66(10):3-8.

24. Report MW. Prevalence of Autism Spectrum Disorder Among Children Aged 8 Years - Autism and Developmental Disabilities Monitoring Network, 11 Sites, United States, 2014. 2018;67(6):1-23.

25. Pinto RNM, Torquato IMB, Collet N, Reichert AP da S, Souza Neto VL de, Saraiva AM. Autismo infantil: impacto do diagnóstico e repercussões nas relações familiares. Rev Gaúcha Enferm [Internet]. [acesso em Out 2018];37(3):1-9.

26. Report MW. Prevalence and Characteristics of Autism Spectrum Disorder Among Children Aged 8 Years--Autism and Developmental Disabilities Monitoring Network, 11 Sites, United States, 2012. 2016; 65(3):1-23.

27. Güngör NK. Overweight and obesity in children and adolescents. J Clin Res Pediatr Endocrinol. 2014;6(3):129-43. doi: 10.4274/Jcrpe.1471.

28. Pimenta TA, Rocha R. A obesidade infantil no Brasil: um estudo comparativo entre a PNSN/1989 e a POF/2008-09 entre crianças de 5 a 9 anos de idade. FIEP Bull Online [Internet]. 2012; 82(1) [acessado em 2018 Out 2]. Disponível em: http://www.fiepbulletin.net/index.php/fiepbulletin/article/view/224.

29. Phillips KL, Schieve LA, Visser S, Boulet S, Sharma AJ, Kogan MD, Boyle CA, Yeargin-allsopp M. Prevalence and impact of unhealthy weight in a national sample of US adolescents with autism and otherlearning and behavioral disabilities. Matern Child Health Journal. v. 18, n. 8, p. 1964-1975, 2014.

30. Khalife N, Kantomaa M, Glover V, Tammelin T, Laitinen J, Ebe-ling H, et al. Childhood attention-deficit/hyperactivity disordersymptoms are risk factors for obesity and physical inacti-vity in adolescence. J Am Acad Child Adolesc Psychiatry.2014;53:425---3.

31. Tyler CV, Schramm SC, Karafa M, Tang AS, Jain AK. Chronic Disease Risks in Young Adults With Autism Spectrum Disorder: Forewarned Is Forearmed. American Journal on Intellectual and Developmental Disabilities. v. 116, n. 5, p. 371-380, 2011.

32. Castro K, Faccioli LS, Baronio D, Gottfried C, Perry IS, Riesgo R. Feeding behavior and dietary intake of male children and adolescents with autism spectrum disorder: A case-control study. Int J Dev Neurosci. 2016;53:68-74.

33. Emidio PP, Fagundes GE, Barchinski MC, Silva MA. Avaliac, ão nutricional em portadores da síndrome autística. Nutrire. 2009;34:382.

34. Zuckerman KE, Hill AP, Guion K, Voltolina L, Fombonne E. Overweight and obesity: prevalence and correlates in a large clinical sample of children with autism spectrum disorder. J Autism Dev Disord. 2014;44:1708---19.

35. Al-Kindi NM, Al-Farsi YM, Waly MI, Al-Shafaee MS, Bakheit CS, Al-Sharbati $\mathrm{MM}, \mathrm{Al}$-Adawi S. Comparative assessment of eating behavior among children 
with autism to typically developing children in Oman. Canad J Clin Nutr 2016; 4 (2): 51-64.

36. Volkert VM, Vaz PC. Recent Studies on Feeding Problems in Children With Autism. J Appl Behav Anal [Internet]. 2010[acesso em 2018 Nov 2];43(1):155-9. Disponivel em: http://www.ncbi.nlm.nih.gov/pmc/PMC2831447.

37. Gray HL, Chiang HM. Brief Report: Mealtime Behaviors of Chinese American Children with Autism Spectrum Disorder. J Autism Dev Disord. Springer US; 2017;47(3):892-7.

38. Cermak SA, Curtin C, Bandini LG. Food selectivity and sensory sensitivity in children with autism spectrum disorders. J Am Diet Assoc. 2010;110(2):238-46.

39. Hsiao EY. Gastrointestinal issues in autism spectrum disorder. Harvard Review of Psychiatry. 2014;22(2):104-111.

40. Chaidez V, Hansen RL, Hertz-Picciotto I. Gastrointestinal problems in children with autism, developmental delays or typical development. J Autism Dev Disord. 2014;44(5):1117-27.

41. Suarez MA, Nelson NW, Curtis AB. Longitudinal follow-up of factors associated with food selectivity in children with autism spectrum disorders. Autism: the international journal of research and practice. 2013;18(8):924-32.

42. Attlee A, Kassem H, Hashim M, Obaid RS. Physical Status and Feeding Behavior of Children with Autism. Indian Journal Pediatrics. 2015;82(8):682-7. 